\title{
Atresia anal grau IV em cão - relato de caso
}

\section{Fabiana Bernardes Almeida Santos}

Médica Veterinária autônoma (Medicina Veterinária do UBM, Barra Mansa, RJ, Brasil) fabi bernardes@yahoo.com.br.

\section{Hellen Magela Barreto}

Médica Veterinária autônoma (Medicina Veterinária do UBM, Barra Mansa, RJ, Brasil) hmagelabarreto@gmail.com.

\section{Simone Pontes Xavier Salles}

Médica Veterinária e Doutora em Medicina Veterinária pela Universidade Federal Rural do Rio de Janeiro (UFRRJ), docente e coordenadora do curso de Medicina Veterinária do UBM. simonesallesvet@hotmail.com.

\section{Alexandre Soares Fagundes \\ Médico Veterinário e Mestre em pela Universidade Federal Rural do Rio de Janeiro (UFRRJ), docente do curso de Medicina Veterinária do UBM. alevetrural@yahoo.com.br.}

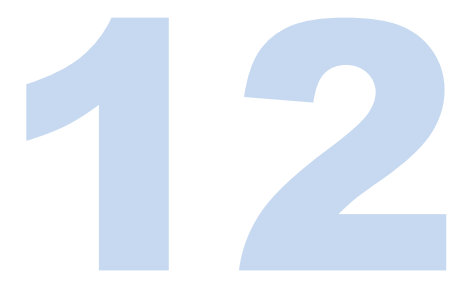

\section{cão -}




\section{Resumo}

Atresia anal consiste em uma anormalidade congênita rara que ocorre em filhotes, sendo incompatível com a vida quando não tratada. Caracteriza-se pela persistência da membrana anal durante a formação embriológica, e pode ser classificada em quatro tipos (graus I, II, III e IV). No início, os sinais podem ser imperceptíveis devido à limpeza constante da mãe sobre os filhotes. O diagnóstico é realizado através de exames clínicos e complementares, como radiografia e ultrassonografia, em que se pode determinar o estágio da enfermidade. O prognóstico é variável, dependendo do grau da anomalia, da precocidade do diagnóstico e do estado geral do paciente. $\mathrm{O}$ presente artigo relata um caso de atresia anal grau IV, associada à fístula retovaginal em um cão da raça Poodle, fêmea, com 30 dias de vida. $O$ animal foi submetido à intervenção cirúrgica para correção do defeito, onde obteve-se sucesso.

Palavras-chave: Anormalidade congênita. Fístula retovaginal. Ânus imperfurado.

\section{Abstract}

Anal atresia consists of a rare congenital abnormality that occurs in puppies, being incompatible with life if left untreated. It is characterized by persistent anal membrane during embryological development and can be classified into four grades (grades I, II, III and IV). At first, the signs may be noticeable due to the constant cleaning of the mother of the puppies. The diagnosis is performed by clinical and laboratory tests, such as X-ray and ultrasound, which can determine the stage of the disease. The prognosis is variable, depending on the degree of the disease, on the early diagnosis and patient's general condition. This article reports a case of grade IV anal atresia associated with rectovaginal fistula in a dog Poodle breed, female, with 30 days of life. The animal underwent surgery to correct the defect, which was obtained success.

Keywords: Congenital abnormality. Rectovaginal fistula. Imperforate anus. 


\section{Introdução}

A atresia anal é a anomalia congênita mais comum em suínos e bezerros, com relatos em potros e rara em cães e gatos, porém, dentro dessa classe de enfermidades, se destaca como a mais frequente em cães. Habitualmente é nomeada como ânus imperfurado e define-se como a persistência da membrana anal durante a formação embrionária (NODEN; LAHUNTA, 1990). A verdadeira incidência dessa anormalidade é de difícil determinação, e pode ser maior do que é relatada, porque muitos filhotes recém-nascidos são submetidos à eutanásia antes de serem avaliados, com base na hipótese de que a correção cirúrgica não possui bom prognóstico. Além disso, dados não publicados podem esconder a exata prevalência de complicações associadas com a cirurgia de correção desta deformidade (PRASSINOS, et. al, 2003; MAHLER e WILLIAMS, 2005; VIANNA e TOBIAS, 2005).

Vianna e Tobias (2005) sugerem que a atresia anal é mais comum em fêmeas que em machos (fêmeas / machos = 1.79/1). Os sinais clínicos podem variar de acordo com o grau da doença, incluindo: tenesmo, ausência de fezes, perda da vitalidade, megacólon, passagem de fezes através da vulva ou uretra, assim como eritema perivulvar, anorexia, dilatação abdominal e saliência perianal (PRASSINOS, et. al, 2003; HEDLUND, 2005). Quatro tipos anatômicos de atresia anal têm sido descritos em cães e gatos (VIANNA e TOBIAS, 2005; ARONSON, 2003; ELLISON e PAPAZOGLOU, 2011), conforme demonstrado no quadro 1.

Quadro 1. Diferentes graus de atresia anal e suas definições

\begin{tabular}{|c|c|}
\hline GRAU & DEFINIÇÃO \\
\hline I & $\begin{array}{r}\text { Há persistência de uma membrana anal; estenose congênita de um ânus } \\
\text { patente }\end{array}$ \\
\hline II & $\begin{array}{r}\text { Há oclusão por uma membrana anal, porém o reto termina imediatamente } \\
\text { cranial ao ânus imperfurado, como uma bolsa cega }\end{array}$ \\
\hline III & $\begin{array}{r}\text { Presença de um ânus imperfurado, e o reto termina como uma bolsa cega } \\
\text { mais cranialmente dentro do canal pélvico }\end{array}$ \\
\hline IV & $\begin{array}{c}\text { O reto cranial termina como bolsa cega no interior do canal pélvico, e há } \\
\text { umanicação persistente entre o reto e a vagina (fêmeas) ou uretra } \\
\text { (machos) }\end{array}$ \\
\hline
\end{tabular}

Fonte: Adaptado de Vianna e Tobias (2005); Aronson (2003); Ellison e Papazoglou (2011) 
SANTOS, F. B. A. et al. Atresia anal grau IV em cão - relato de caso. R. Cientifica UBM - Barra Mansa (RJ), ano XXII, v. 19, n. 36, 1. Sem. 2017. p. 220-228.

ISSN 1516-4071

Repetidamente associada a defeitos como atresia anal, a fístula urorretal tratase de uma anomalia no desenvolvimento do septo urorretal que possibilita a comunicação entre o reto (cólon descendente) e algum dos derivados do seio uretral (NODEN; LAHUNTA, 1990). Em fêmeas, a fístula conecta a parede dorsal da vagina com a parte ventral do reto terminal, que finaliza em bolsa cega. Nos machos essas fístulas se localizam entre o reto e a uretra. Noden e Lahunta (1990) afirmam ainda que estas fístulas são compatíveis com longos períodos de sobrevivência, principalmente em fêmeas, ainda que sejam frequentes os processos infecciosos devido à presença de fezes na uretra e vestíbulo.

O diagnóstico é basicamente clínico, com a anamnese e observação da anomalia durante a inspeção. Pode-se fazer uso da radiografia contrastada ou ultrassonografia para diferenciação dos graus da doença e como um norte para melhor conduta cirúrgica a ser adotada (WHYKES; OLSON, 2003). O tratamento consiste em intervenção cirúrgica, sendo que, na maioria dos casos, o prognóstico é desfavorável, e a mortalidade cirúrgica elevada, já que a maioria desses animais são muito jovens e estão em más condições físicas, o que aumenta o risco de morte do paciente durante o procedimento operatório e anestésico (ARONSON, 2003; SILVA et. al, 2008).

\section{Relato do Caso}

Um cão, fêmea, da raça Poodle, com 30 dias de vida e peso de 1,4kg, foi encaminhado à Clínica Veterinária de Pequenos Animais do Centro Universitário de Barra Mansa.

Durante a anamnese, a proprietária relatou que o animal apresentava dor ao defecar, fezes amolecidas e fétidas, polifagia, oligúria e adipsia. Ao exame clínico, observou-se ausência da abertura anal, dilatação abdominal e eliminação de fezes pela vagina. Diante desse quadro, foi estabelecido o diagnóstico de atresia anal grau IV, associada a fístula retovaginal. Parâmetros vitais como temperatura corporal, frequências cardíaca e respiratória, perfusão capilar e hidratação foram avaliados previamente e se encontravam dentro da normalidade para a espécie.

Em relação à triagem cirúrgica, foi realizado, inicialmente, hemograma completo (Tabela 1), que detectou anemia normocítica. Foi realizado exame ultrassonográfico, 
SANTOS, F. B. A. et al. Atresia anal grau IV em cão - relato de caso. R. Científica UBM - Barra Mansa (RJ), ano XXII, v. 19 , n. 36, 1. Sem. 2017. p. 220-228.

ISSN 1516-4071

que identificou a presença da porção caudal do reto terminando em bolsa cega no interior do canal pélvico, com comunicação persistente entre o reto e a vagina, caracterizando a atresia anal como grau IV.

Tabela 1. Resultado do hemograma completo realizado durante a triagem cirúrgica

\begin{tabular}{c|c|c}
\hline Série Vermelha & Resultado & Valores de referência \\
\hline Hematócrito & $26 \%$ & $37-55 \%$ \\
\hline Eritrócito & $5.650 \mathrm{~mm}^{3}$ & $5.500 .000-8.500 .000 / \mathrm{mm}^{3}$ \\
\hline Proteína Plasmática Total & $7,2 \mathrm{~g} / \mathrm{dl}$ & $6-8 \mathrm{~g} / \mathrm{dL}$ \\
\hline Série Branca & - & - \\
\hline Leucócitos Totais & $7.200 \mathrm{~mm}^{3}$ & $6.000-17.000 / \mathrm{mm}^{3}$ \\
\hline Bastões & 0 & $0-300 / \mathrm{mm}^{3}$ \\
\hline Segmentados & $3.800 \mathrm{~mm}^{3}$ & $3.000-11.500 / \mathrm{mm}^{3}$ \\
\hline Eosinófilos & $130 \mathrm{~mm}^{3}$ & $100-1.250 / \mathrm{mm}^{3}$ \\
\hline Basófilos & 0 & $\mathrm{Raros}$ \\
\hline Linfócitos & $2.300 \mathrm{~mm}^{3}$ & $1.000-4.800 / \mathrm{mm}^{3}$ \\
\hline Monócitos & $830.000 \mathrm{~mm}^{3}$ & $150-1.350 / \mathrm{mm}^{3}$ \\
\hline
\end{tabular}

Valores de referência: Kaneko et al. (1997); Jain (1993).

Após tricotomia da região perianal e abdominal, o paciente foi encaminhado ao centro cirúrgico. Foi utilizada anestesia endovenosa com Cloridrato de Xilazina $(1 \mathrm{mg} / \mathrm{kg})$, Cloridrato de Cetamina $(5 \mathrm{mg} / \mathrm{kg})$ e Diazepam $(0,5 \mathrm{mg} / \mathrm{kg})$. O animal foi mantido em decúbito esternal, a vulva foi ocluída com algodão para que não houvesse contato com fezes (Figura 1).

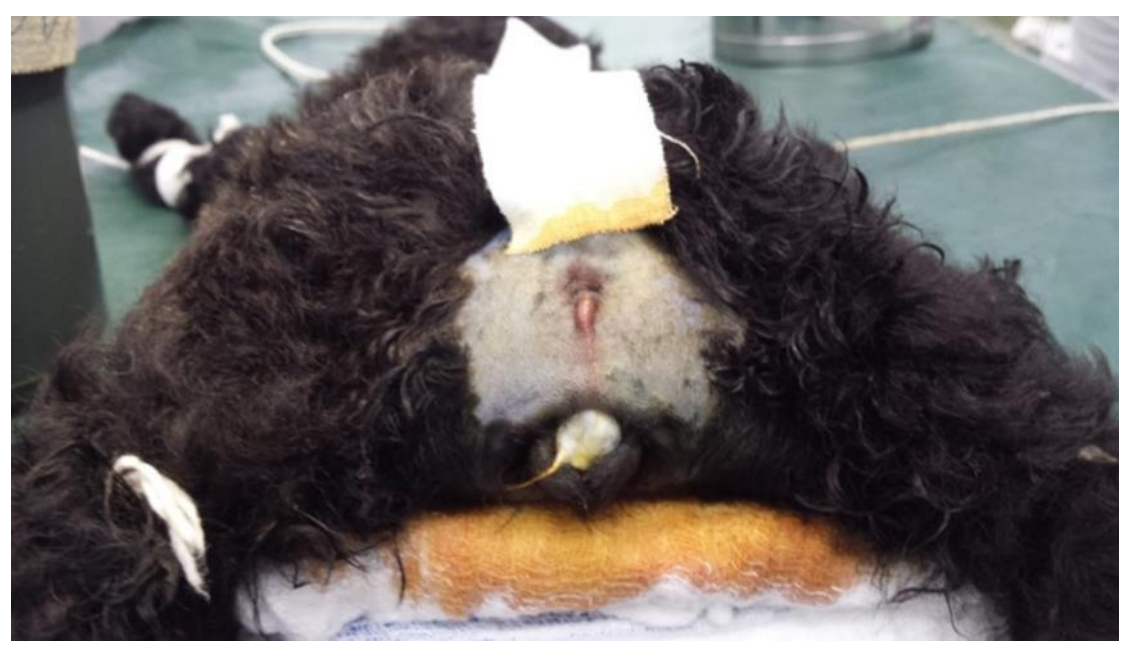

Figura 1. Cão em decúbito esternal para realização do procedimento cirúrgico 
Iniciou-se o procedimento cirúrgico com uma incisão na região anal, em formato de cruz. Não foi possível identificar a porção final do reto e sua comunicação com a vagina, sendo assim, optou-se por realizar a incisão na região abdominal, na linha alba, atingindo os músculos transverso do abdômen, oblíquo externo e oblíquo interno, ultrapassando o peritônio (Figura 2).

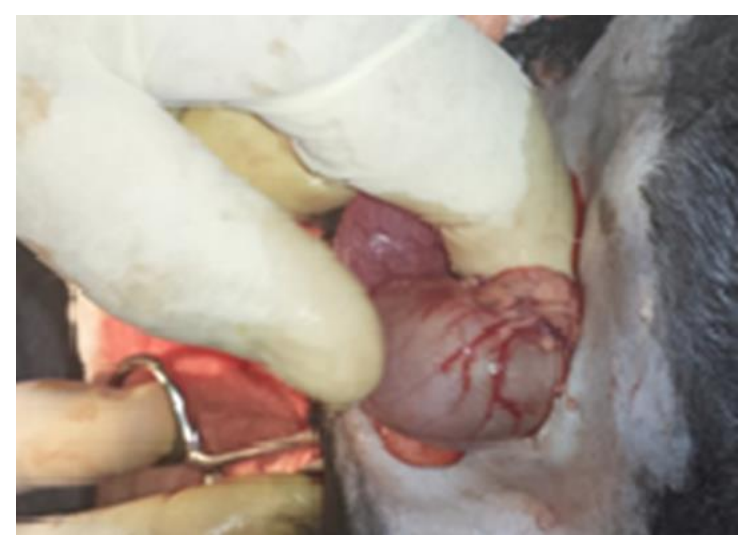

Figura 2. Manobra que demonstra a tração cranial do intestino grosso

Visando localizar a porção final do intestino grosso e sua comunicação com a vagina, foi realizada manobra, tracionando-o caudalmente. Após sua localização, foi realizada a rafia do abdômen com sutura festonada na musculatura e a do peritônio com fio inabsorvível agulhado de Nylon 4-0, com agulha $3 / 8$ de $20 \mathrm{~mm}$ traumática, sutura tipo Cushing no tecido subcutâneo, utilizando-se fio agulhado absorvível Catgut cromado 3-0 com agulha atraumática $3 / 8$ de $20 \mathrm{~mm}$, e pontos simples separados em pele, com fio inabsorvível agulhado de Nylon 4-0 com agulha 3/8 de 20mm traumática. Com uma incisão no local, pôde- se observar a diferenciação de mucosas (Fig. 3).

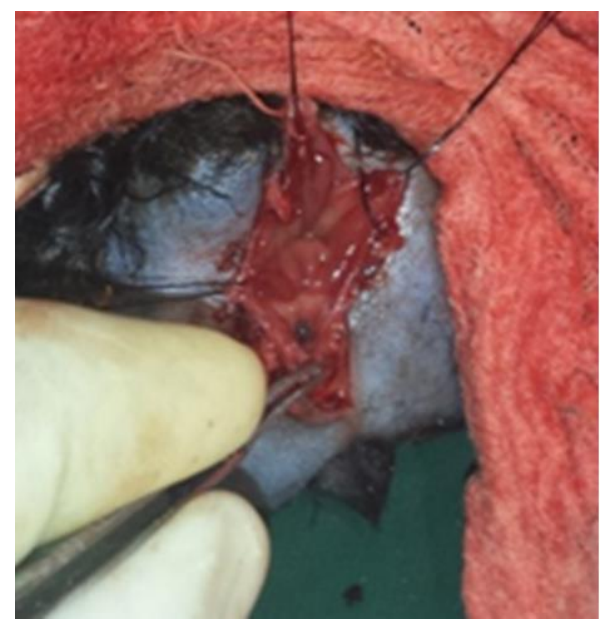

Figura 3. Demonstração da diferença entre a mucosa vaginal e retal. 
Após separar as estruturas reto e vagina, a comunicação retovaginal foi suturada com ponto simples separado, e fio agulhado de nylon 4-0 com agulha 3/8 de $20 \mathrm{~mm}$ de diâmetro traumática. $\mathrm{O}$ reto foi tracionado para o local da incisão inicial, onde sua abertura caudal foi suturada sobre a pele com ponto simples separado e fio agulhado de nylon 4-0 com agulha 3/8 de $20 \mathrm{~mm}$ de diâmetro traumática (figura 4).

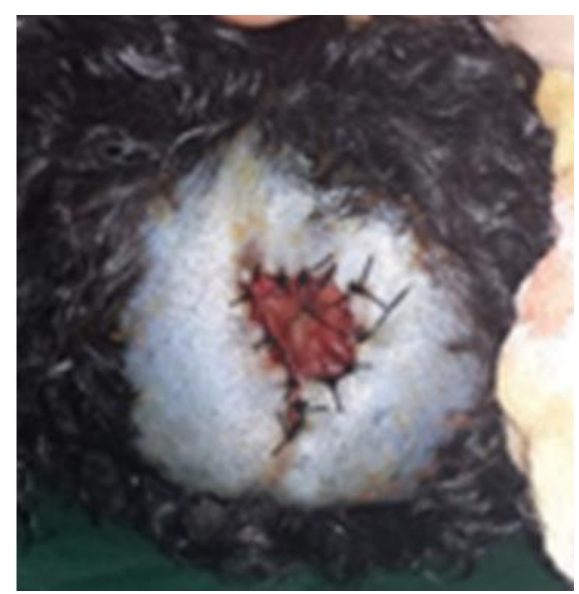

Figura 4. Término da intervenção, com evidenciação da sutura do novo orifício anal.

Durante o tratamento pós-cirúrgico, foram utilizados os seguintes medicamentos: antibiótico (Cefalexina 125mg, 12/12h, durante sete dias), antiinflamatório não esteroidal (Cetoprofeno $5 \mathrm{mg}, 1 / 4$ do comprimido, 24/24h, durante três dias) e Buscopan Gotas (1 gota, 6/6h, durante três dias). Para auxiliar no processo cicatricial, foi realizada aplicação tópica de Rifocina Spray ${ }^{\circledR}$ (Sanofi-Aventis Farmacêutica, São Paulo, Brasil) no local da ferida cirúrgica. Durante esse período, a alimentação foi baseada no fornecimento de ração pastosa conforme instruções do fabricante (Recovery ${ }^{\circledR}$, Royal Canin, Aimargues, França). Sete dias após o procedimento, foi realizada a remoção dos pontos cirúrgicos, e constatou-se que o processo cicatricial foi adequado.

O paciente retornou à clínica 38 dias após a realização do procedimento apresentando o dobro do peso, com $2.800 \mathrm{~g}$ e em perfeitas condições de saúde. A dieta permanece pastosa, pois, segundo a tutora, quando a paciente se alimentava de ração seca, tornava-se evidente a dificuldade da mesma em evacuar. 
SANTOS, F. B. A. et al. Atresia anal grau IV em cão - relato de caso. R. Cientifica UBM- Barra Mansa (RJ), ano XXII, v. 19 , n. 36, 1. Sem. 2017. p. 220-228.

ISSN 1516-4071

\section{Discussão}

De acordo com Aronson (2003) e Silva et al. (2008), o prognóstico da atresia anal, principalmente grau IV é desfavorável e a mortalidade cirúrgica elevada, isso se dá pelos pacientes serem muito jovens e apresentarem más condições físicas, o que aumenta os riscos anestésicos e cirúrgicos. Porém, esse caso descrito foi um sucesso até a presente data.

O exame ultrassonográfico foi decisivo para estabelecimento do tratamento cirúrgico. Através da imagem obtida, pode-se determinar que se tratava do grau IV da patologia, segundo a classificação de Aronson (2003), Ellison e Papazoglou (2011); Hedlund (2005), Vianna e Tobias (2005).

O procedimento cirúrgico foi complexo, devido à necessidade de se realizar uma abertura abdominal para se obter o melhor acesso à fistula, o que também foi relatado por Aronson (2003).

\section{Considerações finais}

Apesar do prognóstico desfavorável da atresia anal grau IV, a cirurgia apresentou resultado satisfatório, culminando com a completa recuperação do paciente. Foi importante para o resultado positivo do procedimento identificar precocemente a patologia, pois evitou maior debilidade física do paciente que poderia levar a complicações durante o trans ou pós operatório.

\section{Referências}

ARONSON, L. Rectum and anus. In: Textbook of small animal surgery, D. Slatter, (Ed), 682-708, Saunders, ISBN 0721686079, Philadelphia, USA. 2003.

ELLISON, G.; PAPAZOGLOU, L. Long-term results of surgery for atresia ani with or without anogenital malformations in puppies and a kitten: 12 cases. Journal of the American Veterinary Medical Association, v. 240, n. 2, p. 186-192, 2011. 
SANTOS, F. B. A. et al. Atresia anal grau IV em cão - relato de caso. R. Cientifica UBM - Barra Mansa (RJ), ano XXII, v. 19 , n. 36, 1. Sem. 2017. p. 220-228.

HEDLUND, C.S. Cirurgia do sistema digestório. In: FOSSUM, T.W. Cirurgia de Pequenos Animais. 2. ed. São Paulo: Roca, 2005, cap. 21p. 277-450.

JAIN, N.C. Essentials of veterinary hematology. Philadelphia: Lea e Febiger, 1993. KANEKO, J.J.; HARVEY, J.W.; BRUSS, M.L. (eds.) Clinical biochemistry of domestic animals. $5^{\text {th }}$ ed. New York: Academic Press, 1997.

MAHLER, S.; WILLIAMS, G. Preservation of the fistula for reconstruction of the anal canal and the anus in atresia ani and rectovestibular fistula in 2 dogs. Veterinary Surgery, v. 34, n. 2, p. 148-152, 2005.

NODEN, D.M; LAHUNTA, A. Embriologia de los animales domésticos: mecanismo de desarrollo y malformaciones. Espanha; Acribia, 1990.

PRASSINOS, N., PAPAZOGLOU, L., ADAMAMA-MORAITOU, K., GALATOS, A., GOULETSOU, P.; RALLIS, T. Congenital anorectal abnormalities in six dogs. Veterinary record, v. 153, n. 3, p. 81-85, 2003.

SILVA, C. M.; SAKAMOTO, S. S.; FERREIRA, G. T. N. M. et al. Estudo retrospectivo dos casos de atresia anal em cães atendidos no hospital veterinário de Araçatuba no período de 2001-2008. Veterinária e Zootecnia. v. 15, p. 38, 2008.

VIANNA, M.; TOBIAS, K. Atresia ani in the dog: a retrospective study. Journal of the American Animal Hospital Association, v. 41, n. 5, p. 317-322, 2005.

WHYKES P.M.; OLSON O.N, 2003. Vagina, vestibule and vulva. In: Slatter D.H (Ed) Textbook of small animal surgery. $3^{\text {rd }}$ ed. Philadelphia : WB Saunders, pp1502-1510. 